\title{
EFFECTS OF CONFIGURATION INTERACTION FOR DIELECTRONIC RECOMBINATION OF Na-LIKE IONS FORMING Mg-LIKE IONS
}

\author{
D.-H. KwON ${ }^{1}$ AND D. W. SAVIN \\ Columbia Astrophysics Laboratory, Columbia University, New York, NY 10027, USA \\ Received 2011 January 29; accepted 2011 March 24; published 2011 May 17
}

\begin{abstract}
Theoretical dielectronic recombination (DR) rate coefficient calculations can be sensitive to configuration interaction (CI) between resonances with different captured electron principle quantum numbers $n$. Here we explore the importance of this multi- $n$ CI process for DR via $2 l \rightarrow 3 l^{\prime}$ core excitations and its effect on the total DR rate coefficient. Results are presented for selected Na-like ions from $\mathrm{Ca}^{9+}$ to $\mathrm{Zn}^{19+}$. We find that including this multi- $n$ CI can reduce the DR rate coefficient by up to $\sim 10 \%$ at temperatures where an ion is predicted to form in collisional ionization equilibrium and up to $\sim 15 \%$ at higher temperatures. To a first approximation, this will translate into a corresponding increase in the ion abundance. Charge state distributions calculation seeking to be accurate to better than $10 \%$ will thus need to take this effect into account. We also present simple fits to the calculated rate coefficients for ease of incorporation into plasma models.
\end{abstract}

Key words: atomic data - atomic processes

\section{INTRODUCTION}

Dielectronic recombination (DR) is the dominant electronion recombination process for most atomic ions in cosmic plasmas, whether they be photoionized or collisionally ionized (Ferland et al. 1998; Kallman \& Palmeri 2007). As a result, reliable DR data are critical for the analysis, modeling, and interpretation of astrophysical spectra. To meet this need atomic physicists have been and continue to carry out theoretical and experimental DR studies (e.g., Badnell et al. 2003; Gu 2004; Beiersdorfer 2003; Schippers 2009; Schippers et al. 2010).

$\mathrm{DR}$ is a two-step recombination process which begins when a free electron collides with an ion of charge $q+$ in an initial state $i$. The incident electron collisionally excites a core electron of the ion with principal quantum number $n_{\mathrm{c}}$ and is simultaneously captured, forming a system of state $j$. This process is known as dielectronic capture. The energy of the intermediate system lies in the continuum and it may autoionize. DR occurs when the state $j$ radiatively decays to a bound state $f$ by emitting a photon $h v$ which reduces the total energy of the recombined system to below its ionization threshold, resulting in a bound system.

Recently, in Kwon \& Savin (2011), we have investigated the cause for the discrepancy between theory and experiment for the simple $\mathrm{M}$-shell ion Na-like $\mathrm{Fe}^{15+}$ forming $\mathrm{Mg}$-like $\mathrm{Fe}^{14+}$ via $\Delta n_{\mathrm{c}}=1$ core excitation of a $2 l$ electron. We demonstrated in that work the importance of configuration interaction (CI) between resonances with different captured electron principal quantum numbers $n$ and found that this multi- $n$ CI can lead to significant reduction in DR resonance strength for resonances where $n \geqslant 5$.

Here we investigate the effects of this multi- $n$ CI on the total Maxwellian DR rate coefficients required to model cosmic plasmas. We present results for selected Na-like ions of elements with even proton number $Z$ from $\mathrm{Ca}^{9+}$ to $\mathrm{Zn}^{19+}$. The rest of this paper is organized as follows. In Section 2, we describe the calculational approach used to obtain total DR rate coefficients. Calculated total DR rate coefficients are shown for the selected ions and compared with those of single- $n$ CI in Section 3.

\footnotetext{
1 Current address: Laboratory for Quantum Optics, Korea Atomic Energy Research Institute, Daejeon 305-600, Republic of Korea.
}

Section 4 discusses the reduction of the total DR rate coefficient due to multi- $n \mathrm{CI}$ and the $Z$ dependence of the effect. Lastly, we summarize our results in Section 5.

\section{CALCULATIONAL METHOD}

We have used the flexible atomic code (FAC) of Gu (2008) to calculate the required atomic parameters as detailed in Kwon \& Savin (2011). Here we briefly review those calculations as applied here to generate rate coefficients.

For $\Delta n_{\mathrm{c}}=0$ and 1 core excitations of a $3 l$ electron, we included the same autoionization and radiative decay channels as those of $\mathrm{Gu}$ (2004) who also performed FAC calculations for Na-like ions. Initial radial wave functions were optimized on the $2 l^{8} 3 l^{\prime} 3 l^{\prime \prime}$ configuration group of the recombined ion. CI for the resonance state was considered only within the same $n$ complex (i.e., single- $n \mathrm{CI}$ ). The resonance energies for the $\Delta n_{\mathrm{c}}=0$ excitations were adjusted so that the various series limits matched the corresponding excitation energies of the recombining ion as given by Ralchenko et al. (2008). Explicit calculations for autoionization and radiative decay rates were performed for states up to $n=37$ for $Z<26$ and $n=38$ for $Z \geqslant 26$. Captured electron angular momentums of $l^{\prime \prime \prime} \leqslant 15$ were included. A simple hydrogenic scaling law was used to extrapolate the resonance energies, autoionization rates, and radiative decay rates of the captured electron for higher principal quantum numbers of the valence electron up to $n=1000$. For these higher $n$ levels, the radiative decay rate of the core electron was set to that of the last $n$ level for which explicit calculations were carried out.

For $\Delta n_{\mathrm{c}}=1 \mathrm{DR}$ via inner-shell core excitation of a $2 l$ electron, we performed a large scale, fully relativistic calculation first using only single- $n$ CI up to $n=14$ and then with multi$n$ CI between all $2 l^{7} 3 l^{\prime} 3 l^{\prime \prime} n l^{\prime \prime \prime}$ complexes from $n=3$ to 14 . For higher $n$ up to 1000 , we calculated resonance energies, autoionization rates, and radiative rates using the extrapolation method described above. All possible core configurations were considered for $l^{\prime \prime \prime} \leqslant 5$. For both approaches, we included $2 s \rightarrow$ $3 l$ promotions which were not considered in the theoretical works of Gu (2004) or Altun et al. (2006). 


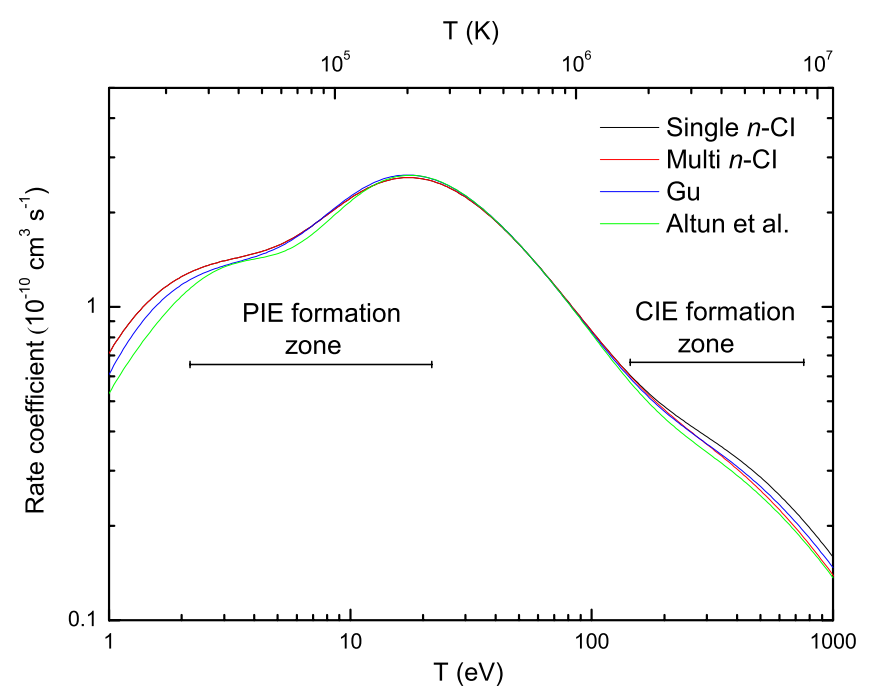

Figure 1. Total DR rate coefficient for ground state $\mathrm{Fe}^{15+}$ forming $\mathrm{Fe}^{14+}$ versus temperature $T$. The black line shows our present FAC results for single- $n$ CI and the red curve including multi- $n \mathrm{CI}$ for $2 l \rightarrow 3 l^{\prime}$ core excitations. The blue and green lines show, respectively, the FAC results of Gu (2004) and the AUTOSTRUCTURE results of Altun et al. (2006), which are both single- $n$ $\mathrm{CI}$ calculations. The horizontal bars show where $\mathrm{Fe}^{15+}$ is predicted for form in photoionization equilibrium (PIE; Kallman et al. 2004) and in collisional ionization equilibrium (CIE; Bryans et al. 2009).

Initial radial wave functions were optimized on the $2 l^{8} 3 l^{\prime} 3 l^{\prime \prime}$ configuration of the recombined ion as was also done for $\Delta n_{\mathrm{c}}=0$ and 1 core excitations of a $3 l$ electron. We considered the $2 l^{8} 3 l^{\prime}, 2 l^{8} n l^{\prime \prime \prime}$, and $2 l^{7} 3 l^{\prime} 3 l^{\prime \prime}$ autoionization channels, and the $2 l^{8} 3 l^{\prime} 3 l^{\prime \prime}$ and $2 l^{8} 3 l^{\prime} n l^{\prime \prime \prime}$ radiative channels for both core and captured electron transitions. For sufficiently high $n$, the $2 l^{8} 3 l^{\prime} n l^{\prime \prime \prime}$ levels lie in the continuum and radiative Decays to Autoionizing levels followed by radiative Cascades (DAC) can take place. These channels were included here and are available for $n \geqslant 5$ for $\mathrm{Ca}^{9+}, n \geqslant 6$ for $\mathrm{Ti}^{11+}$ and $\mathrm{Cr}^{13+}$, and $n \geqslant 7$ for $\mathrm{Fe}^{15+}, \mathrm{Ni}^{17+}$, and $\mathrm{Zn}^{19+}$. As shown in Kwon \& Savin (2011), multi- $n$ CI results in the opening up of additional autoionization and radiative decay channels.

The total DR rate coefficient averaged over a MaxwellBoltzmann electron energy distribution at temperature $T$ is given by (Shore 1969)

$$
\alpha_{i}(T)=\frac{1}{2 g_{i}}\left(\frac{4 \pi a_{0}^{2} R y}{k_{\mathrm{B}} T}\right)^{3 / 2} \sum_{j} g_{j} A_{j i}^{a} B_{j} \exp \left(-\frac{E_{i j}}{k_{\mathrm{B}} T}\right),
$$

where $g_{i}$ and $g_{j}$ are the statistical weights of the states $i$ and $j$, $E_{i j}$ is the resonance energy, $A_{j i}^{a}$ is the autoionization rate from the state $j$ to $i, R y$ is the Rydberg energy, $k_{\mathrm{B}}$ is the Boltzmann constant, and $a_{0}$ is the Bohr radius. $B_{j}$ denotes the radiative stabilizing branching ratio and can be expressed as (Behar et al. 1996)

$$
B_{j}=\frac{\sum_{t} A_{j t}^{r}+\sum_{t^{\prime}} A_{j t^{\prime}}^{r} B_{t^{\prime}}}{\sum_{k} A_{j k}^{a}+\sum_{f} A_{j f}^{r}},
$$

where the final states $t$ and $t^{\prime}$ are below and above the ionization threshold, respectively. $B_{t^{\prime}}$ is the branching ratio for radiative stabilization of $t^{\prime}$ and can be determined by evaluating $B_{j}$ iteratively.

\section{RESULTS}

Total DR rate coefficients for ground state $\mathrm{Fe}^{15+}$ are shown in Figure 1. Note first our fully single- $n$ CI results which we

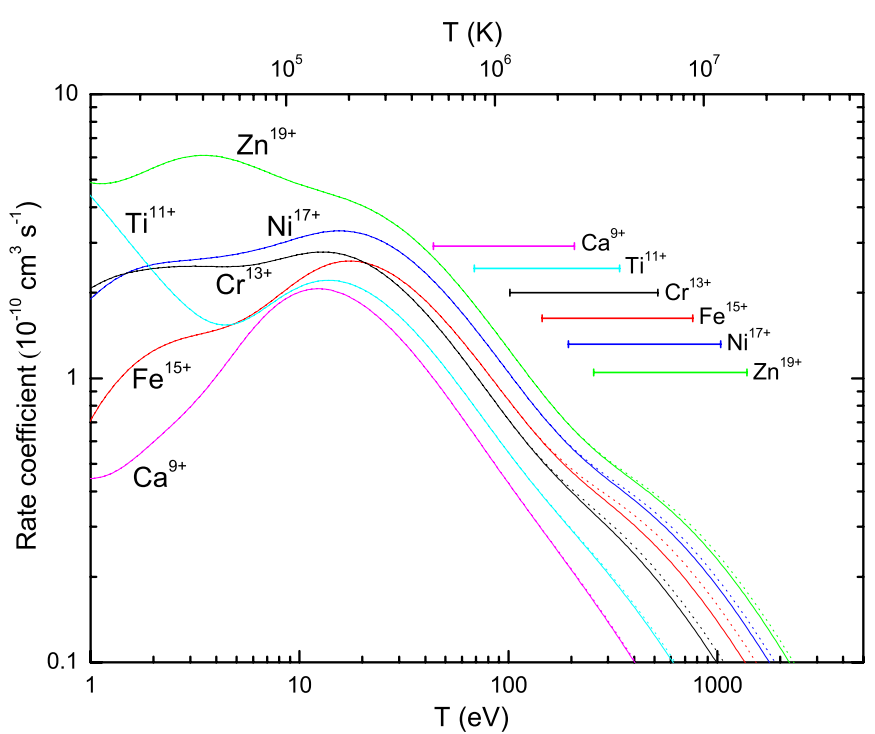

Figure 2. Total DR rate coefficients for various Na-like ions forming Mg-like ions. The solid line represents our results including $2 l \rightarrow 3 l^{\prime}$ multi- $n \mathrm{CI}$ result and the dotted line shows our fully single- $n$ CI results. The CIE temperature regime for each ion from Bryans et al. (2009) is given by the horizontal bars.

show for comparison to the also fully single- $n$ CI works of $\mathrm{Gu}$ (2004) and Altun et al. (2006). The differences at temperatures where $\mathrm{Fe}^{15+}$ is predicted to form in photoionization equilibrium (PIE; Kallman et al. 2004) are attributed to the well-known problem of accurately calculating low-energy DR resonances (Schippers et al. 2004; Schmidt et al. 2008; Fu et al. 2008). Here the difference is largely due to the various predicted resonance energies for the $2 l^{8} 3 l^{\prime} 4 l^{\prime \prime} 4 l^{\prime \prime \prime}$ resonances.

At temperatures where $\mathrm{Fe}^{15+}$ is predicted to form in collisional ionization equilibrium (CIE; Bryans et al. 2009), our single- $n$ CI results are about $8 \%$ larger than those of Gu (2004). We have verified that this is a result of the $2 s \rightarrow 3 l$ core excitations which were not accounted for by $\mathrm{Gu}$ (2004). Our results are also about 15\% larger than those of Altun et al. (2006). We attribute this as being due, in part, to their not accounting for $2 s \rightarrow 3 l$ core excitations.

Figure 1 also shows our results including multi- $n$ CI for $2 l \rightarrow 3 l^{\prime}$ core excitations. The resulting rate coefficient is about $13 \%$ smaller at CIE temperatures than our fully single- $n$ CI results. This is due to the reduction in resonance strengths as detailed in Kwon \& Savin (2011).

Figure 2 shows the total rate coefficients for $\mathrm{Ca}^{9+}, \mathrm{Ti}^{11+}, \mathrm{Cr}^{13+}$, $\mathrm{Fe}^{15+}, \mathrm{Ni}^{17+}$, and $\mathrm{Zn}^{19+}$. Our results are shown for fully single- $n$ $\mathrm{CI}$ as well as when multi- $n \mathrm{CI}$ for $2 l \rightarrow 3 l^{\prime}$ core excitations is included. The total rate coefficient at CIE temperatures decreases as a result of this multi- $n$ CI. This decrease grows with increasing $Z$ from $\mathrm{Cr}^{9+}$ to $\mathrm{Fe}^{15+}$ and then decreases with $Z$ from $\mathrm{Fe}^{15+}$ to $\mathrm{Zn}^{19+}$. This can be most clearly seen in Figure 3 which shows our results including $2 s \rightarrow 3 l$ multi- $n$ CI compared to our fully single- $n$ CI results. Rate coefficient can be reduced by up to $10 \%$ at CIE temperatures and by up to $15 \%$ at even higher temperatures.

For convenience in plasma modeling, we have fitted our total DR rate coefficients including $2 l \rightarrow 3 l^{\prime}$ multi- $n$ CI. We use the formula

$$
\alpha(T)=\left(k_{\mathrm{B}} T\right)^{-3 / 2} \sum_{i} c_{i} \exp \left(-\frac{E_{i}}{k_{\mathrm{B}} T}\right)
$$


Table 1

Fit Parameters $c_{i}\left(10^{-10} \mathrm{~cm}^{3} \mathrm{~s}^{-1} \mathrm{eV}^{3 / 2}\right)$ and $E_{i}(\mathrm{eV})$ for Our Total DR Rate Coefficients Including $2 l \rightarrow 3 l^{\prime}$ Multi- $n$ CI of Selected Na-like Ions Forming Mg-like Ions

\begin{tabular}{|c|c|c|c|c|c|c|c|c|}
\hline Ion & $c_{1}$ & $c_{2}$ & $c_{3}$ & $c_{4}$ & $c_{5}$ & $c_{6}$ & $c_{7}$ & $c_{8}$ \\
\hline $\mathrm{Ca}^{9+}$ & $3.688 \mathrm{E}-2$ & $5.331 \mathrm{E}-1$ & $7.542 \mathrm{E}-1$ & $1.588 \mathrm{E}+1$ & $5.193 \mathrm{E}+1$ & $3.730 \mathrm{E}+2$ & $1.062 \mathrm{E}+2$ & $6.774 \mathrm{E}+2$ \\
\hline $\mathrm{Ti}^{11+}$ & $2.357 \mathrm{E}+0$ & $2.514 \mathrm{E}+0$ & $4.756 \mathrm{E}+0$ & $2.266 \mathrm{E}+1$ & $4.109 \mathrm{E}+2$ & $1.833 \mathrm{E}+2$ & $2.549 \mathrm{E}+2$ & $1.465 \mathrm{E}+3$ \\
\hline $\mathrm{Cr}^{13+}$ & $3.118 \mathrm{E}-1$ & $3.327 \mathrm{E}+0$ & $5.040 \mathrm{E}+1$ & $1.241 \mathrm{E}+1$ & $1.448 \mathrm{E}+2$ & $4.497 \mathrm{E}+2$ & $6.256 \mathrm{E}+2$ & $3.338 \mathrm{E}+3$ \\
\hline $\mathrm{Fe}^{15+}$ & $1.514 \mathrm{E}-1$ & $2.664 \mathrm{E}+0$ & $1.013 \mathrm{E}+1$ & $2.623 E+2$ & $2.871 \mathrm{E}+1$ & $7.396 \mathrm{E}+2$ & $5.407 \mathrm{E}+3$ & $7.760 \mathrm{E}+2$ \\
\hline $\mathrm{Ni}^{17+}$ & $4.285 \mathrm{E}-1$ & $2.590 \mathrm{E}+1$ & $1.805 \mathrm{E}+0$ & $1.037 \mathrm{E}+2$ & $4.659 \mathrm{E}+2$ & $7.993 \mathrm{E}+2$ & $1.336 \mathrm{E}+3$ & $7.786 \mathrm{E}+3$ \\
\hline \multirow[t]{2}{*}{$\mathrm{Zn}^{19+}$} & $1.850 \mathrm{E}+0$ & $4.088 \mathrm{E}+0$ & $3.002 \mathrm{E}+1$ & $1.714 \mathrm{E}+2$ & $5.198 \mathrm{E}+2$ & $1.037 \mathrm{E}+3$ & $2.017 \mathrm{E}+3$ & $1.061 \mathrm{E}+4$ \\
\hline & $E_{1}$ & $E_{2}$ & $E_{3}$ & $E_{4}$ & $E_{5}$ & $E_{6}$ & $E_{7}$ & $E_{8}$ \\
\hline$\overline{\mathrm{Ca}^{9+}}$ & $1.475 \mathrm{E}-1$ & $6.127 \mathrm{E}-1$ & $2.457 \mathrm{E}+0$ & $5.611 \mathrm{E}+0$ & $1.309 \mathrm{E}+1$ & $2.247 \mathrm{E}+1$ & $9.534 \mathrm{E}+1$ & $3.244 \mathrm{E}+2$ \\
\hline $\mathrm{Ti}^{11+}$ & $1.329 \mathrm{E}-1$ & $7.599 \mathrm{E}-1$ & $1.430 \mathrm{E}+0$ & $7.797 \mathrm{E}+0$ & $2.249 \mathrm{E}+1$ & $3.881 \mathrm{E}+1$ & $1.725 \mathrm{E}+2$ & $4.298 \mathrm{E}+2$ \\
\hline $\mathrm{Cr}^{13+}$ & $5.928 \mathrm{E}-1$ & $1.338 \mathrm{E}+0$ & $7.102 \mathrm{E}+0$ & $2.535 \mathrm{E}+0$ & $1.751 \mathrm{E}+1$ & $1.902 \mathrm{E}+2$ & $3.287 \mathrm{E}+1$ & $5.396 \mathrm{E}+2$ \\
\hline $\mathrm{Fe}^{15+}$ & $1.312 \mathrm{E}+0$ & $2.077 \mathrm{E}+0$ & $3.456 \mathrm{E}+0$ & $2.130 \mathrm{E}+1$ & $7.395 \mathrm{E}+0$ & $3.846 \mathrm{E}+1$ & $6.624 \mathrm{E}+2$ & $2.307 \mathrm{E}+2$ \\
\hline $\mathrm{Ni}^{17+}$ & $2.669 \mathrm{E}-1$ & $3.183 \mathrm{E}+0$ & $1.279 \mathrm{E}+0$ & $1.067 \mathrm{E}+1$ & $2.573 \mathrm{E}+1$ & $4.747 \mathrm{E}+1$ & $2.985 E+2$ & $7.946 \mathrm{E}+2$ \\
\hline $\mathrm{Zn}^{19+}$ & $2.212 \mathrm{E}-1$ & $7.110 \mathrm{E}-1$ & $3.175 \mathrm{E}+0$ & $7.108 \mathrm{E}+0$ & $2.682 \mathrm{E}+1$ & $5.278 \mathrm{E}+1$ & $3.487 \mathrm{E}+2$ & $9.334 \mathrm{E}+2$ \\
\hline
\end{tabular}

Table 2

Same as Table 1 but with $c_{i}$ in units of $\mathrm{cm}^{3} \mathrm{~s}^{-1} \mathrm{~K}^{3 / 2}$ and $T_{i}$ in $\mathrm{K}$

\begin{tabular}{|c|c|c|c|c|c|c|c|c|}
\hline Ion & $c_{1}$ & $c_{2}$ & $c_{3}$ & $c_{4}$ & $c_{5}$ & $c_{6}$ & $c_{7}$ & $c_{8}$ \\
\hline $\mathrm{Ca}^{9+}$ & $4.610 \mathrm{E}-6$ & $6.664 \mathrm{E}-5$ & $9.429 \mathrm{E}-5$ & $1.985 \mathrm{E}-3$ & $6.492 \mathrm{E}-3$ & $4.663 \mathrm{E}-2$ & $1.328 \mathrm{E}-2$ & $8.469 \mathrm{E}-2$ \\
\hline $\mathrm{Ti}^{11+}$ & $2.946 \mathrm{E}-4$ & $3.143 \mathrm{E}-4$ & $5.946 \mathrm{E}-4$ & $2.833 \mathrm{E}-3$ & $5.137 \mathrm{E}-2$ & $2.292 \mathrm{E}-2$ & $3.187 \mathrm{E}-2$ & $1.832 \mathrm{E}-1$ \\
\hline $\mathrm{Cr}^{13+}$ & $3.898 \mathrm{E}-5$ & $4.159 \mathrm{E}-4$ & $6.301 \mathrm{E}-3$ & $1.551 \mathrm{E}-3$ & $1.811 \mathrm{E}-2$ & $5.622 \mathrm{E}-2$ & $7.820 \mathrm{E}-2$ & $4.174 \mathrm{E}-1$ \\
\hline $\mathrm{Fe}^{15+}$ & $1.893 \mathrm{E}-5$ & $3.330 \mathrm{E}-4$ & $1.266 \mathrm{E}-3$ & $3.279 \mathrm{E}-2$ & $3.590 \mathrm{E}-3$ & $9.246 \mathrm{E}-2$ & $6.760 \mathrm{E}-1$ & $9.701 \mathrm{E}-2$ \\
\hline $\mathrm{Ni}^{17+}$ & $5.357 \mathrm{E}-5$ & $3.238 \mathrm{E}-3$ & $2.256 \mathrm{E}-4$ & $1.296 \mathrm{E}-2$ & $5.824 \mathrm{E}-2$ & $9.993 \mathrm{E}-2$ & $1.670 \mathrm{E}-1$ & $9.734 \mathrm{E}-1$ \\
\hline \multirow[t]{2}{*}{$\mathrm{Zn}^{19+}$} & $2.312 \mathrm{E}-4$ & $5.111 \mathrm{E}-4$ & $3.753 \mathrm{E}-3$ & $2.143 \mathrm{E}-2$ & $6.498 \mathrm{E}-2$ & $1.296 \mathrm{E}-1$ & $2.522 \mathrm{E}-1$ & $1.327 \mathrm{E}+0$ \\
\hline & $T_{1}$ & $T_{2}$ & $T_{3}$ & $T_{4}$ & $T_{5}$ & $T_{6}$ & $T_{7}$ & $T_{8}$ \\
\hline $\mathrm{Ca}^{9+}$ & $1.712 \mathrm{E}+3$ & $7.111 \mathrm{E}+3$ & $2.851 \mathrm{E}+4$ & $6.511 \mathrm{E}+4$ & $1.519 \mathrm{E}+5$ & $2.608 \mathrm{E}+5$ & $1.106 \mathrm{E}+6$ & $3.765 \mathrm{E}+6$ \\
\hline $\mathrm{Ti}^{11+}$ & $1.542 \mathrm{E}+3$ & $8.819 \mathrm{E}+3$ & $1.659 \mathrm{E}+4$ & $9.048 \mathrm{E}+4$ & $2.610 \mathrm{E}+5$ & $4.503 E+5$ & $2.002 \mathrm{E}+6$ & $4.988 \mathrm{E}+6$ \\
\hline $\mathrm{Cr}^{13+}$ & $6.879 \mathrm{E}+3$ & $1.553 \mathrm{E}+4$ & $8.242 \mathrm{E}+4$ & $2.942 \mathrm{E}+4$ & $2.032 \mathrm{E}+5$ & $2.207 \mathrm{E}+6$ & $3.814 \mathrm{E}+5$ & $6.262 \mathrm{E}+6$ \\
\hline $\mathrm{Fe}^{15+}$ & $1.522 \mathrm{E}+4$ & $2.411 \mathrm{E}+4$ & $4.010 \mathrm{E}+4$ & $2.471 \mathrm{E}+5$ & $8.581 \mathrm{E}+4$ & $4.463 \mathrm{E}+5$ & $7.687 \mathrm{E}+6$ & $2.677 \mathrm{E}+6$ \\
\hline $\mathrm{Ni}^{17+}$ & $3.098 \mathrm{E}+3$ & $3.693 \mathrm{E}+4$ & $1.484 \mathrm{E}+4$ & $1.239 \mathrm{E}+5$ & $2.986 \mathrm{E}+5$ & $5.508 \mathrm{E}+5$ & $3.464 \mathrm{E}+6$ & $9.221 \mathrm{E}+6$ \\
\hline $\mathrm{Zn}^{19+}$ & $2.567 \mathrm{E}+3$ & $8.250 \mathrm{E}+3$ & $3.685 E+4$ & $8.249 \mathrm{E}+4$ & $3.112 \mathrm{E}+5$ & $6.125 \mathrm{E}+5$ & $4.047 \mathrm{E}+6$ & $1.083 \mathrm{E}+7$ \\
\hline
\end{tabular}

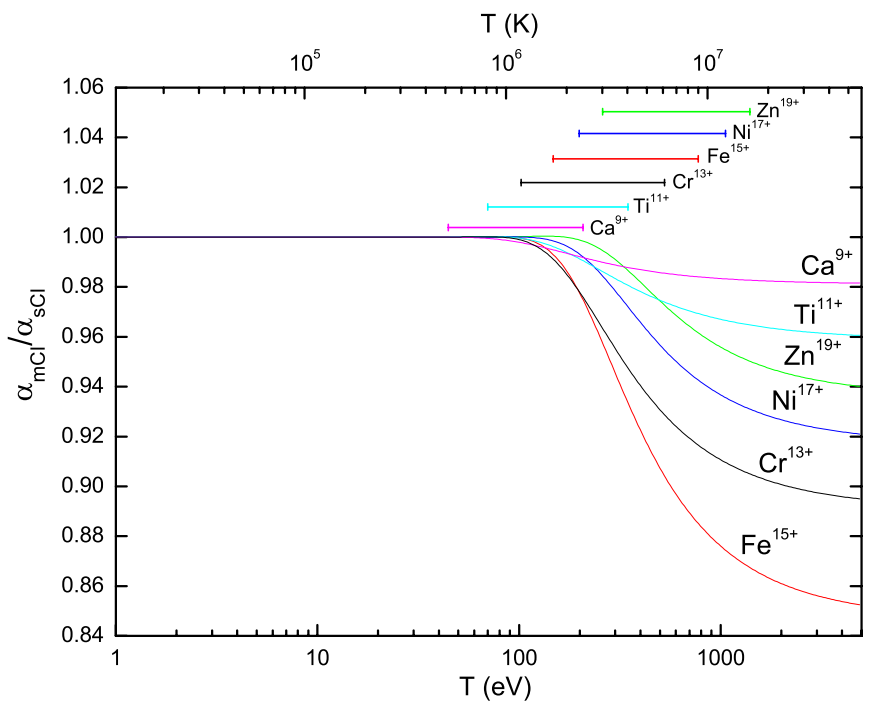

Figure 3. Ratio of our total DR rate coefficients including $2 l \rightarrow 3 l^{\prime}$ multi- $n \mathrm{CI}$ to our fully single- $n \mathrm{CI}$ calculations for various $\mathrm{Na}$-like ions forming $\mathrm{Mg}$-like ions. The CIE temperature regime for each ion from Bryans et al. (2009) is given by the horizontal bars.

where $T$ is in units of $\mathrm{K}$. Table 1 lists the fitted parameters. These fits are accurate to better than $\approx 3.5 \%$ from $0.1 \mathrm{eV}$ to $5 \mathrm{keV}$ for $\mathrm{Ca}^{9+}, \mathrm{Ti}^{11+}, \mathrm{Ni}^{17+}$, and $\mathrm{Zn}^{19+}$. For $\mathrm{Cr}^{13+}$ the accuracy is better than $\approx 3.5 \%$ from $0.43 \mathrm{eV}$ to $5 \mathrm{keV}$ and within $10 \%$ from $0.1 \mathrm{eV}$ to $0.43 \mathrm{eV}$. For $\mathrm{Fe}^{15+}$ the accuracy is better than $\approx 3.5 \%$ from
$0.85 \mathrm{eV}$ to $5 \mathrm{keV}$ and within $\approx 23.5 \%$ from $0.1 \mathrm{eV}$ to $0.85 \mathrm{eV}$. Equation (3) can also be re-expressed as

$$
\alpha(T)=T^{-3 / 2} \sum_{i} c_{i} \exp \left(-\frac{T_{i}}{T}\right) .
$$

The fit parameters for this are listed in Table 2 and the accuracies are the same as those given above.

\section{DISCUSSION}

The charge state distribution of electron-ionized gas in coronal equilibrium, where three-body recombination, dust, and radiation can all be ignored, is determined by the balance of electron impact ionization with DR and radiative recombination. In CIE, these rates balance and one finds (Hahn et al. 2011)

$$
\frac{n_{q+1}}{n_{q}}=\frac{\alpha_{\mathrm{I}}^{q}}{\alpha_{\mathrm{R}}^{q+1}}
$$

Here, $n_{q}$ and $n_{q+1}$ are the densities of the $q$ and $q+1$ charge states, respectively, $\alpha_{\mathrm{I}}^{q}$ is the ionization rate coefficient for charge state $q$, and $\alpha_{\mathrm{R}}^{q+1}$ is the electron-ion recombination rate coefficient for charge state $q+1$. In CIE, recombination is due primarily to DR (Bryans et al. 2006, 2009). Thus, it is immediately clear that a $10 \%$ reduction in the DR rate coefficient corresponds to a similar increase in ratio of the charge states. Charge state distribution calculations seeking to be accurate to 
better than $10 \%$ will thus need to take the effect of multi- $n \mathrm{CI}$ into account.

The $Z$ dependency of the rate coefficient reduction due to multi- $n$ CI can be explained by the competition of two effects which scale differently with $Z$. The amount of configuration mixing $\Delta^{2}$ between two interacting levels is given by (Cowan 1981)

$$
\Delta^{2}=\frac{\left(R^{\mathrm{k}}\right)^{2}}{\left(E_{2}-E_{1}\right)^{2}},
$$

where $R^{\mathrm{k}}$ denotes the CI Coulomb radial integral in units of energy and $E_{2}$ and $E_{1}$ are the energies of two interacting levels. For the $(N+1)$-electron intermediate resonance state, $R^{k}$ is proportional to $Z-S$ which is the actual nuclear charge $Z$ minus the effective screening charge $S$ of the $N$ electrons as seen by the Rydberg electron. $E_{2}-E_{1}$ is proportional to $(Z-S)^{2}$. So, $\Delta^{2}$ varies as $(Z-S)^{-2}$ (Cowan 1981) and the importance of multi- $n$ CI decreases with $Z$.

Multi- $n$ CI has little effect on the $n=3$ resonances because their overlap in energy with higher $n$ resonances is rare. That is not the case for $n \geqslant 4$ resonances of $\mathrm{Ca}^{9+}, \mathrm{Ti}^{11+}$, and $\mathrm{Cr}^{13+}$ and for $n \geqslant 5$ resonances of $\mathrm{Fe}^{15+}, \mathrm{Ni}^{17+}$, and $\mathrm{Zn}^{19+}$. The strength of these resonances relative to the non-overlapping low $n$ resonances grows with $Z$. Thus, their contribution to the plasma rate coefficient grows with $Z$. However, the strengths of the overlapping resonances are reduced by multi- $n$ CI. The amount of this reduction decreases with $Z$ as explained above. It is the combination of the increasing importance of the overlapping resonances with the decreasing importance of CI, both increasing with $Z$, which leads to the behavior seen in Figures 2 and 3.

\section{SUMMARY}

We have presented results showing the effects on DR rate coefficients due to $\mathrm{CI}$ between resonances with different captured electron quantum number $n$ for DR via $2 l \rightarrow 3 l^{\prime}$ core excitation. Data have been presented for selected Na-like ions from $\mathrm{Ca}^{9+}$ to $\mathrm{Zn}^{19+}$. This multi- $n \mathrm{CI}$ can reduce the DR rate coefficient at CIE temperatures by up to $\sim 10 \%$ and by up to $\sim 15 \%$ at higher temperatures. To a first approximation this will translate into a corresponding increase in the ion abundance. Charge state distributions calculation seeking to be accurate to better than $10 \%$ will thus need to take this effect into account. We have also presented simple fitting formulae so that our DR rate coefficients can be readily incorporated into plasma models.

We thank M. Hahn for providing the fits to our results. This work was supported in part by the NASA Astronomy and Physics Research and Analysis (APRA) Program and the NASA Solar and Heliospheric Physics Supporting Research and Technology Program.

\section{REFERENCES}

Altun, Z., Yumak, A., Badnell, N. R., Loch, S. D., \& Pindzola, M. S. 2006, A\&A, 447, 1165

Badnell, N. R., et al. 2003, A\&A, 409, 1151

Behar, E., Mandelbaum, P., Schwob, J. L., Bar-Shalom, A., Oreg, J., \& Goldstein, W. H. 1996, Phys. Rev. A, 54, 3070

Beiersdorfer, P. 2003, ARA\&A, 41, 343

Bryans, P., Badnell, N. R., Gorczyca, T. W., Laming, J. M., Mitthumsiri, W., \& Savin, D. W. 2006, ApJS, 167, 343

Bryans, P., Landi, E., \& Savin, D. W. 2009, ApJ, 691, 1540

Cowan, R. D. 1981, The Theory of Atomic Structure and Spectra (Berkeley: Univ. California Press)

Ferland, G. J., Korista, K. T., Verner, D. A., Ferguson, J. W., Kingdon, J. B., \& Verner, E. M. 1998, PASP, 110, 761

Fu, J., Gorczyca, T. W., Nikolic, D., Badnell, N. R., Savin, D. W., \& Gu, M. F. 2008, Phys. Rev. A, 77, 032713

Gu, M. F. 2004, ApJS, 153, 389

Gu, M. F. 2008, Can. J. Phys., 86, 675

Hahn, M., et al. 2011, ApJ, 729, 76

Kallman, T. R., \& Palmeri, P. 2007, Rev. Mod. Phys., 79, 79

Kallman, T. R., Palmeri, P., Bautista, M. A., Mendoza, C., \& Krolik, J. H. 2004, ApJS, 155, 675

Kwon, D.-H., \& Savin, D. W. 2011, Phys. Rev. A, 83, 012701

Ralchenko, Yu., Kramida, A. E., Reader, J., \& NIST ASD Team 2008, NIST Atomic Spectra Database, Version 3.1.5 (Gaithersburg: NIST), http://physics.nist.gov/asd3

Schippers, S. 2009, J. Phys.: Conf. Ser., 163, 012001

Schippers, S., Lestinsky, M., Müller, A., Savin, D. W., Schmidt, E. W., \& Wolf, A. 2010, Int. Rev. At. Mol. Phys., 1, 109

Schippers, S., Schnell, M., Brandau, C., Kieslich, S., Müller, A., \& Wolf, A. 2004, A\&A, 421, 1185

Schmidt, E. W., et al. 2008, A\&A, 492, 265

Shore, B. W. 1969, ApJ, 158, 1205 